\title{
Ehrenfest-time dependence of weak localization in open quantum dots
}

\author{
Saar Rahav ${ }^{1}$ and Piet W. Brouwer ${ }^{1}$ \\ ${ }^{1}$ Laboratory of Atomic and Solid State Physics, Cornell University, Ithaca 14853, USA.
}

\begin{abstract}
Semiclassical theory predicts that the weak localization correction to the conductance of a ballistic chaotic cavity is suppressed if the Ehrenfest time exceeds the dwell time in the cavity [I. L. Aleiner and A. I. Larkin, Phys. Rev. B 54, 14424 (1996)]. We report numerical simulations of weak localization in the open quantum kicked rotator that confirm this prediction. Our results disagree with the 'effective random matrix theory' of transport through ballistic chaotic cavities.
\end{abstract}

PACS numbers: 73.23.-b,05.45.Mt,05.45.Pq,73.20.Fz

The wave nature of electrons is the cause of striking effects that are absent in classical mechanics. The best known manifestations of quantum mechanics on electrical transport are weak localization (WL), universal conductance fluctuations (UCF), and shot noise [1]. In this letter we consider such quantum mechanical effects in a ballistic cavity with chaotic classical dynamics. A phenomenological theory of shot noise, weak localization, and conductance fluctuations in a ballistic chaotic cavity is given by random matrix theory [2]. The predictions of random matrix theory have been confirmed using semiclassical methods [3, 4, 5, 6].

Since they are intrinsically quantum effects, it is legitimate to ask what happens to shot noise, weak localization, and conductance fluctuations if the classical limit is taken. The classical limit corresponds to the case that the electron wavelength is much smaller than the system size. The relevant time scale for this question is the socalled "Ehrenfest time" 3, 7], the minimal time at which the wave nature of electrons become apparent. This is the time at which a minimal wavepacket is stretched to the system size $L$ (or the size of the lead opening). It is given by

$$
\tau_{\mathrm{E}}=\lambda^{-1} \ln k L,
$$

where $\lambda$ is the Lyapunov exponent characterizing the chaotic motion in the cavity, and $k$ the electron wavenumber.

The effects of the Ehrenfest time are most prominent if $\tau_{\mathrm{E}}$ is larger than the mean dwell time in the cavity $\tau_{\mathrm{D}}$. In this regime most of the transport is classical and shot noise is suppressed 8, 9, 10]. The effect of $\tau_{\mathrm{E}}$ on WL was first addressed by Aleiner and Larkin [3], who predicted a suppression proportional to $\exp \left(-2 \tau_{\mathrm{E}} / \tau_{\mathrm{D}}\right)$. Exponential suppression of WL, but with a different exponent, was also found by Adagideli [11]. Suppression of WL was observed experimentally by Yevtushenko et al. 12]. There is no semiclassical theory of the Ehrenfest-time dependence of UCF.

Numerical simulation of systems with large Ehrenfest times is difficult due to the need for (exponentially) high values of $k L$. To reduce numerical costs Jacquod et al. proposed to replace the two-dimensional cavity by a one-

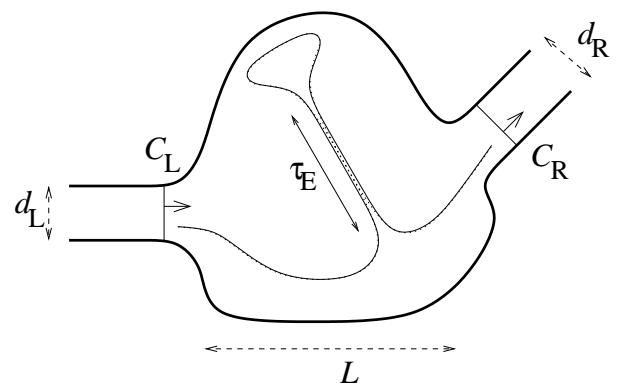

FIG. 1: Schematic picture of a ballistic chaotic cavity of size $L$. The cavity is attached to two leads, labeled "L" and "R", and with width $d_{\mathrm{L}}$ and $d_{\mathrm{R}}$, respectively. The contours $C_{\mathrm{L}}$ and $C_{\mathrm{R}}$ drawn in the leads are used for the calculation of conductance. A schematic drawing of relevant trajectories for weak localization is depicted inside the cavity. (The physical trajectories scatter from the cavity walls many times.)

dimensional quantum map [13]. The map is 'opened', so that simulation of transport properties is possible. An example of such a map, the open kicked rotator, was used to obtain numerical results for shot noise 14], WL 15], and UCF 16, 17, 18]. The simulation results for shot noise were in agreement with semiclassical predictions [8]. In contrast, the results for WL and UCF are remarkably different: no dependence on $\tau_{\mathrm{E}}$ was found. To explain their results, the authors of Refs. 15, 16, 17, 18] proposed an 'effective random matrix theory': Noting that quantum diffraction contributes only if the classical dwell time is larger than $\tau_{\mathrm{E}}$, they proposed to apply random matrix theory only to the part of phase space with dwell times longer than $\tau_{\mathrm{E}}[19]$. Since UCF and WL are not dependent on the size of the phase space involved, the 'effective random matrix' theory is able to describe the numerical results for WL and UCF [20].

Apart from the prediction of the size of the weak localization correction, the 'effective random matrix theory' and the semiclassical theory have different predictions of the minimal time required for weak localization to occur. In the semiclassical theory, quantum interference requires a minimal wavepacket to be split and reunited, which takes a minimal time $2 \tau_{\mathrm{E}}$. A schematic diagram drawing relevant semiclassical trajectories for WL is de- 
picted in Fig. 10 This is different in the 'effective random matrix theory, where quantum interference is fully established already after a time $\tau_{\mathrm{E}}$.

It is the purpose of this letter to resolve the discrepancy between the reported numerical simulations and the semiclassical theory for weak localization. In the first part of this letter, we review the original semiclassical theory [3] of the Ehrenfest time dependence of WL and amend it in order to correctly implement classical correlations in an open cavity 21]. In the second half we report time-resolved numerical simulations of weak localization in the open quantum kicked rotator. The time-resolved simulations allow us to determine the time at which WL first appears, as well as the effect of a finite Ehrenfest time on the conductance. We find that the time-resolved simulations for WL and the corrected semiclassical theory are consistent.

Semiclassical theory. The semiclassical theory of Ref. 3 considers a two-dimensional chaotic cavity which is coupled to two electron reservoirs via contacts of widths $d_{\mathrm{L}, \mathrm{R}}$, see Fig. 1] One has $d_{\mathrm{L}, \mathrm{R}} \ll L$, so that electron dynamics inside the cavity is ergodic. The system is studied in the semiclassical limit, where the number of open channels is large, $d_{\mathrm{L}, \mathrm{R}} k \gg 1$. This separation of length scales is equivalent to the following separation of time scales $(v k)^{-1} \ll L / v \sim 1 / \lambda \ll \tau_{\mathrm{D}}$, where $v$ is the electron velocity, and the dwell time is $\tau_{\mathrm{D}}=\pi \mathcal{A} /\left(d_{\mathrm{L}}+d_{\mathrm{R}}\right) v$, $\mathcal{A}$ being the area of the cavity. It will be useful to define the probability that an electron at a random point will leave the cavity through contact $i, P_{i}=d_{i} /\left(d_{\mathrm{R}}+d_{\mathrm{L}}\right)$.

The central objects in the semiclassical theory of Aleiner and Larkin are the non-oscillating parts of the product of advanced and retarded Green functions [3]. The "diffuson" $\mathcal{D}$ is composed of combinations of orbits with themselves while the "cooperon" $\mathcal{C}$ is composed of orbits and their time reversed counterparts. Quantum effects are introduced by including a weak additional random quantum potential. This random potential leads to two different effects: phase space diffusion, and coupling between paths which are close in phase space. Both are expected due to quantum uncertainty. After averaging over the random potential, the equation of motion for the leading order diffuson, $\mathcal{D}^{0}$, is 3 ]

$$
\left[-i \omega+\hat{L}_{1}-\gamma_{\mathrm{q}} \frac{\partial^{2}}{\partial \phi_{1}^{2}}\right] \mathcal{D}^{0}(\omega ; 1,2)=\delta(1,2),
$$

where $j \equiv\left(\phi_{j}, \mathbf{R}_{j}\right)=1,2$ denotes the phase space coordinates, limited to the energy shell: $\mathbf{R}$ is the electron position while the angle $\phi$ is the direction of its velocity $\mathbf{v}$. The strength of the phase space diffusion is $\gamma_{\mathrm{q}} \simeq \lambda^{2} / k v$. The operator $\hat{L}_{1}$ is the Liouville operator. For a hard wall cavity, $\hat{L}_{1}=\mathbf{v}_{1} \cdot \partial / \partial \mathbf{R}_{1}$, with appropriate boundary conditions at the walls. The symbol $\delta(1,2) \equiv 2 \pi \delta\left(\mathbf{R}_{1}-\mathbf{R}_{2}\right) \delta\left(\phi_{1}-\phi_{2}\right)$ denotes a delta function on the energy shell. The leading order cooperon, $\mathcal{C}^{0}$, also satisfies Eq. (2).
The WL correction to the diffuson arises from combinations of a trajectory with small angle self-intersection and a partner without such self-intersection [3, 22], see Fig. 1] The 'interference region' is composed of close phase space points (with distance $\sim k^{-1 / 2}$ ) where quantum diffraction is important. This leads to a quantum correction for the diffuson $\mathcal{D}=\mathcal{D}^{0}+\Delta \mathcal{D}$ with [3]

$$
\begin{gathered}
\Delta \mathcal{D}(1,2)=\mathcal{D}^{0}(1, \overline{2}) \frac{\mathcal{C}^{0}(\overline{2}, 2)}{2 \pi \hbar \nu}+\frac{\mathcal{C}^{0}(1, \overline{1})}{2 \pi \hbar \nu} \mathcal{D}^{0}(\overline{1}, 2) \\
-\int d 3 \mathcal{D}^{0}(1,3) \mathcal{D}^{0}(\overline{3}, 2)\left[\hat{L}_{3}-\gamma_{\mathrm{q}} \frac{\partial^{2}}{\partial \phi_{3}^{2}}\right] \frac{\mathcal{C}^{0}(3, \overline{3})}{2 \pi \hbar \nu}
\end{gathered}
$$

where $\bar{j}=\left(\phi_{j}+\pi, \mathbf{R}_{j}\right)$ denotes the time reversal of phase space point $j$ and $\nu=m / 2 \pi \hbar^{2}$ is the density of states per unit area. Since we consider DC transport, the frequency $\omega$ has been set to zero.

The total (DC) conductance of the dot can be expressed in terms of the diffuson $\mathcal{D}[\underline{3}, \underline{6}]$,

$$
\begin{aligned}
G= & 2 \pi \hbar \nu v^{2} \int_{C_{\mathrm{R}}} d l_{1} \int_{\phi_{\mathrm{R}}-\pi / 2}^{\phi_{\mathrm{R}}+\pi / 2} \frac{d \phi_{1}}{2 \pi} \cos \left(\phi_{\mathrm{R}}-\phi_{1}\right) \\
& \times \int_{C_{\mathrm{L}}} d l_{2} \int_{\phi_{\mathrm{L}}-\pi / 2}^{\phi_{\mathrm{L}}+\pi / 2} \frac{d \phi_{2}}{2 \pi} \cos \left(\phi_{\mathrm{L}}-\phi_{2}\right) \mathcal{D}(1,2) .
\end{aligned}
$$

Here $G$ is measured in units of the conductance quantum $2 e^{2} / h$, and $C_{\mathrm{L}}$ and $C_{\mathrm{R}}$ denote cross section contours of the contacts. The angle $\phi_{\mathrm{L}}$ is the direction of the inwardpointing normal to $C_{\mathrm{L}}$, while $\phi_{\mathrm{R}}$ is the outward-pointing normal to $C_{\mathrm{R}}$.

The WL correction to the conductance $\delta G$ is obtained by substituting Eq. (3) for $\Delta \mathcal{D}$ into Eq. (4). The first two terms in Eq. (3) contain paths which leave the dot at the point of entry, and hence do not contribute to the transmission. The phase space point 3 in the third term in Eq. (3) can be viewed as the center of the 'interference region' depicted in Fig. 1 As the two diffusons and the cooperon head into opposite directions in configuration space, one can treat them as statistically uncorrelated and estimate them independently [3].

Both the cooperon and the product of diffusons in Eq. (3) exhibit non-trivial correlations [3], since the incoming path is strongly correlated with the outgoing one, cf. Fig. 1. An intuitive way to estimate the cooperon (or the product of diffusons) was presented by Vavilov and Larkin [23]. They demonstrated that the effect of phase space diffusion is equivalent to an average over phase space regions of size $\sim k^{-1 / 2}$ near 3 and $\overline{3}$. Following Ref. 23, we consider phase space points $3^{\prime}$ and $3^{\prime \prime}$ within a distance of order $k^{-1 / 2}$ from 3 . The phase space distance between classical orbits starting at the points $3^{\prime}$, $3^{\prime \prime}$ will diverge exponentially due to the chaotic dynamics and will be macroscopic after a time $\tau_{\mathrm{E}} / 2$. Once the phase space distance between the trajectories is large, they can be considered uncorrelated. 
For the cooperon, the classical correlations mean that it takes a minimal time to form a closed loop. Let us denote by $t_{j}$ the time it takes for a classical trajectory starting at phase space point $j$ to leave the cavity. If $t_{\overline{3}}<\tau_{\mathrm{E}} / 2$ all the points near $\overline{3}$ leave the cavity before it is possible to close a loop. In contrast, when $t_{\overline{3}}>\tau_{\mathrm{E}} / 2$, the cooperon is estimated by propagating phase space points close to $\overline{3}$ forward in time, and points close to 3 backward in time, for a time of $\tau_{\mathrm{E}} / 2$. After this propagation the points which contribute to the local average are uncorrelated. Then the cooperon can be estimated using its ensemble average $\tau_{\mathrm{D}} / \mathcal{A}$. The resulting cooperon in the open cavity is given by

$$
\mathcal{C}^{0}(3, \overline{3})=\left\langle\mathcal{C}^{0}\left(3^{\prime \prime}, \overline{3}^{\prime}\right)\right\rangle \simeq \frac{\tau_{\mathrm{D}}}{\mathcal{A}} \theta\left(t_{\overline{3}}-\tau_{\mathrm{E}} / 2\right),
$$

where $\theta(x)=1$ if $x>0$ and 0 otherwise.

The product of diffusons is estimated similarly. The phase space points 1 and 2 in Eq. (3) refer to two different contacts. If $t_{3}<\tau_{\mathrm{E}} / 2$, trajectories starting close to 3 are still correlated when they leave the cavity. Therefore, they cannot arrive at points on different contacts, leading to a vanishing contribution to $\delta G$. When $t_{3}>\tau_{\mathrm{E}} / 2$ the lead integrals will result in a factor of $P_{R} P_{L}$, as in Refs. 3, 6. Note that the WL paths are composed of four 'legs' with minimal time of $\tau_{\mathrm{E}} / 2$, so that the minimal time of the whole path is $2 \tau_{\mathrm{E}}$.

The resulting WL correction is

$$
\delta G=-P_{\mathrm{R}} P_{\mathrm{L}} \frac{\tau_{\mathrm{D}}}{\mathcal{A}} \int d 3 \theta\left(t_{3}-\tau_{\mathrm{E}} / 2\right) \hat{L}_{3} \theta\left(t_{\overline{3}}-\tau_{\mathrm{E}} / 2\right) .
$$

The Liouville operator in Eq. (6) measures the rate of flow of probability density out of the integration range of the phase space variable $3[3]$. The boundary of the integration range is composed of the lead phase space points propagated backward for a time $\tau_{\mathrm{E}} / 2$. This leaves only a fraction $\exp \left(-\frac{\tau_{\mathrm{E}}}{2 \tau_{\mathrm{D}}}\right)$ of the size of the boundary at the lead. In addition, only points which also have $t_{\overline{3}}>\tau_{\mathrm{E}} / 2$ will have a non-vanishing cooperon, leading to another factor of $\exp \left(-\frac{\tau_{\mathrm{E}}}{2 \tau_{\mathrm{D}}}\right)$. Hence, we find

$$
\delta G=-P_{\mathrm{R}} P_{\mathrm{L}} e^{-\tau_{\mathrm{E}} / \tau_{\mathrm{D}}} .
$$

The result (17) shows that, even after accounting for classical correlations that were not accounted for in Ref. 3 , the WL correction to transmission is suppressed exponentially. However, the exponent is different from that of Ref. 3, where it is reported that $\delta G \propto \exp \left(-2 \tau_{\mathrm{E}} / \tau_{\mathrm{D}}\right)$.

Numerical simulations. We have compared the predictions of the semiclassical theory to numerical simulations of the open quantum kicked rotator. The time evolution of the kicked rotator is discrete, and given in terms of the Floquet operator $\psi(t+1)=\mathcal{F} \psi(t)$. The state of the system $\psi$ is a finite vector of dimension $M$. The Floquet operator is an $M \times M$ matrix and reads

$$
\begin{aligned}
F_{n m}= & M^{-1 / 2} e^{-i \pi / 4+i \pi(m-n)^{2} / M} \\
& \times e^{-i(\kappa M / 4 \pi)(\cos (2 \pi m / M+\theta)+\cos (2 \pi n / M+\theta))},
\end{aligned}
$$

where $\kappa$ is a numerical parameter that determines the classical dynamics of the map and $0 \leq \theta<2 \pi / M$ a parameter that sets the precise quantization condition. The region $\kappa \gtrsim 7.5$ is associated with classically chaotic dynamics. The size of the matrix $M$ is taken to be even.

To open the system, two consecutive sets of $N$ elements of $\psi$ are chosen as the contacts [13]. A scattering matrix is then constructed by the rule

$$
S(\varepsilon)=\sum_{t=1}^{\infty} e^{i \varepsilon t} S(t) ; \quad S(t)=P[\mathcal{F} Q]^{t-1} \mathcal{F} P^{T},
$$

where $\varepsilon$ is the quasienergy, $P$ is a $2 N \times M$ matrix projecting on the contact sites, and $Q \equiv 1-P^{T} P$. The semiclassical limit corresponds to $M \rightarrow \infty$ while keeping $\tau_{\mathrm{D}}=M / 2 N$ fixed. For large values of $\kappa$ the Lyapunov exponent $\lambda$ can be approximated $\lambda \simeq \ln (\kappa / 2)$, yielding

$$
\tau_{\mathrm{E}} \simeq \frac{\ln N}{\ln (\kappa / 2)},
$$

up to an $N$-independent constant. Once the scattering matrix is known, the conductance $G$ follows from the Landauer formula, $G(\varepsilon)=\sum_{m=1}^{N} \sum_{n=N+1}^{2 N}\left|S_{m n}(\varepsilon)\right|^{2}$.

We study transport properties as a function of time, simply by truncating the sum in Eq. (9) after a time $t=t_{0}$. We calculate the ensemble averaged conductance by averaging over $\varepsilon, \theta$, lead positions, and a small range of $\kappa$. An advantage of the time-resolved approach is the ability to evaluate the quasienergy average analytically. In order to achieve sufficient accuracy, we take between 20000 and 80000 realizations for $\theta$, the lead positions, and $\kappa$.

Ideally, for two contacts with $N$ channels, the ensemble average of the classical conductance is $N / 2$ and WL can be calculated by taking the difference between the quantum and classical conductances. In practice, however, the ensemble average of the classical conductance may differ from $N / 2$ and this simple procedure fails. Alternatively, one compares conductances with and without a time-reversal symmetry breaking perturbation. However, such a perturbation may affect the classical dynamics, complicating the interpretation of the results. Instead, we choose to subtract the classical conductance by comparing conductances at $N$ and $2 N$ channels, for the same realization of the classical parameters. The classical conductance is proportional to the channel number, whereas the WL correction is independent of it.

Simulation results for the WL correction to the conductance for $\tau_{\mathrm{D}}=5$ and $\kappa \approx 10$ are shown in Fig. 22 as a function of the cut-off time $t_{0}$. We also performed simulations for $\tau_{\mathrm{D}}=5$ and $\kappa \approx 20, \tau_{\mathrm{D}}=10$ and $\kappa \approx 10$, and $\tau_{\mathrm{D}}=10$ and $\kappa \approx 20$. In all cases, the derivative $d \tau_{\mathrm{E}} / d \ln N$, as extracted from the low- $t_{0}$ part of the curves, is consistent with an onset of the WL correction after a time $2 \tau_{\mathrm{E}}$, in agreement with the predictions of the semiclassical theory. A summary of our results for $\delta G$ is shown in the 


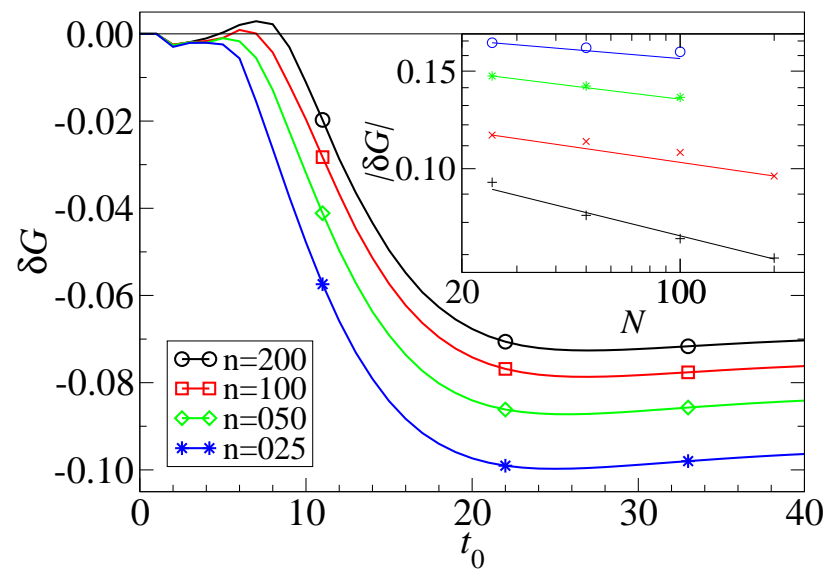

FIG. 2: Time-resolved weak localization correction $\delta G$ as a function of the cut-off time $t_{0}$, for $\kappa \approx 10$ and $\tau_{\mathrm{D}}=5$, and for four values of $N$. Inset: summary of results for the weak localization correction. Data points are obtained from the limit $t_{0} \gg \tau_{\mathrm{D}}$ of simulations for $\tau_{\mathrm{D}}=5$ and $\kappa \approx 10(+)$, $\tau_{\mathrm{D}}=5$ and $\kappa \approx 20(\times), \tau_{\mathrm{D}}=10$ and $\kappa \approx 10(*)$, and $\tau_{\mathrm{D}}=10$ and $\kappa \approx 20$ (o). The solid lines are fits $\propto \exp \left(-\tau_{\mathrm{E}} / \tau_{\mathrm{D}}\right)$, with $d \tau_{\mathrm{E}} / d \ln N$ taken from the low- $t_{0}$ part of the simulation data.

inset of Fig. 21 together with a fit $\propto \exp \left(-\tau_{\mathrm{E}} / \tau_{\mathrm{D}}\right)$, with $d \tau_{\mathrm{E}} / d \ln N$ obtained from the low- $t_{0}$ part of the data. The simulated $\delta G$ has a systematic dependence on $\tau_{\mathrm{E}}$, consistent with the semiclassical theory. Previous numerical calculations of $\delta G$ did not show a dependence on $\tau_{\mathrm{E}}$ [15], probably due to insufficient accuracy. The fact that the over-all magnitude of WL is smaller than in Eq. (7) is well known [15] and caused by a lack of ergodicity for the dwell times we consider. Note that our results for both the onset of WL and its magnitude disagree with the 'effective random matrix theory'. We have further verified our results by calculating $\delta G$ for the three-kick model, a version of the open quantum kicked rotator in which time-reversal symmetry can be broken [15]. We found $\delta G=0$ when the symmetry is broken.

We believe that our simulation results, together with the corrected semiclassical theory, resolves the controversy around the Ehrenfest-time dependence of the weak localization correction to the conductance. Our results quantitatively confirm the corrected semiclassical theory and disagree with the 'effective random matrix theory'. The results presented here are limited to weak localization and do not carry over to conductance fluctuations. We have also carried out numerical simulations of the variance of the conductance for the open quantum kicked rotator 24], and find behavior consistent with that reported previously in the literature [16, 17, 18]: no dependence of conductance fluctuations on the Ehrenfest time $\tau_{\mathrm{E}}$ and an onset time $\tau_{\mathrm{E}}$, not $2 \tau_{\mathrm{E}}$. Currently, there is no semiclassical theory these results can be compared with.
We would like to thank C. Beenakker, S. Fishman, H. Schomerus, P. Silvestrov, and D. Ullmo for discussions. This work was supported by the NSF under grant no. DMR 0334499 and by the Packard Foundation.

[1] C. W. J. Beenakker and H. van Houten, Solid State Physics 44, 1 (1991).

[2] C. W. J. Beenakker, Rev. Mod. Phys. 69, 731 (1997).

[3] I. L. Aleiner and A. I. Larkin, Phys. Rev. B 54, 14423 (1996).

[4] N. Argaman, Phys. Rev. Lett. 75, 2750 (1995).

[5] Y. M. Blanter and E. V. Sukhorukov, Phys. Rev. Lett. 84, 1280 (2000).

[6] Y. Takane and K. Nakamura, J. Phys. Soc. Japan 66, 2977 (1997); 67, 397 (1998).

[7] G. M. Zaslavsky, Phys. Rep. 80, 157 (1981).

[8] O. Agam, I. Aleiner, and A. Larkin, Phys. Rev. Lett. 85, 3153 (2000).

[9] C. W. J. Beenakker and H. van Houten, Phys. Rev. B 43, 12066 (1991).

[10] S. Oberholzer, E. V. Sukhorukov, and C. Schönenberger, Nature 415, 765 (2002).

[11] I. Adagideli, Phys. Rev. B 68, 233308 (2003).

[12] O. Yevtushenko, G. Lütjering, D. Weiss, and K. Richter, Phys. Rev. Lett. 84, 542 (2000). Note, however, that this suppression of WL resulted from competition of $\tau_{\mathrm{E}}$ and the dephasing time $\tau_{\phi}$, not of $\tau_{\mathrm{E}}$ and $\tau_{\mathrm{D}}$.

[13] P. Jacquod, H. Schomerus, and C. W. J. Beenakker, Phys. Rev. Lett. 90, 207004 (2003).

[14] J. Tworzydlo, A. Tajic, H. Schomerus, and C. W. J. Beenakker, Phys. Rev. B 68, 115313 (2003).

[15] J. Tworzydlo, A. Tajic, and C. W. J. Beenakker, Phys. Rev. B 70, 205324 (2004).

[16] P. Jacquod and E. V. Sukhorukov, Phys. Rev. Lett. 92, 116801 (2004).

[17] J. Tworzydlo, A. Tajic, and C. W. J. Beenakker, Phys. Rev. B 69, 165318 (2004).

[18] J. Tworzydlo, A. Tajic, H. Schomerus, P. W. Brouwer, and C. W. J. Beenakker, Phys. Rev. Lett. 93, 186806 (2004).

[19] P. G. Silvestrov, M. C. Goorden, and C. W. J. Beenakker, Phys. Rev. B 67, 241301 (2003).

[20] A 'microscopic theory' showing Ehrenfest-time independent WL was reported in R. S. Whitney and Ph. Jacquod, Phys. Rev. Lett. 94, 116801 (2005). We feel that this theory can not be considered an independent calculation of WL, as it is based on assumptions equivalent to the 'effective random matrix theory'.

[21] We do not consider the later theory of Ref. 11, because the calculational scheme used there does not lead to a unitary scattering matrix. We have verified explicitly that our method to calculate WL preserves unitarity.

[22] M. Sieber and K. Richter, Phys. Scripta T90, 128 (2001).

[23] M. G. Vavilov and A. I. Larkin, Phys. Rev. B 67, 115335 (2003).

[24] S. Rahav and P. W. Brouwer, unpublished (2005). 